\title{
Relação entre a posição Canguru e a estabilidade fisiológica e equilíbrio sono-vigília de recém-nascidos prematuros na UTIN e percepção materna
}

\author{
Relationship between the kangaroo position and \\ physiological stability and sleep-wake balance of \\ premature newborns in the NICU and maternal \\ perception
}

\author{
Karine Souza Andrade Nisi' ${ }^{1}$ \\ Marimar Goretti Andreazza 2 \\ Evellin de Oliveira Gomes ${ }^{3}$ \\ Palmira Donda Soares 4 \\ Arlete Ana Motter 5
}

\begin{abstract}
1,3-5 Secretaria Municipal da Saúde de Curitiba (Curitiba). Paraná, Brasil. karineandrade_nisi@hotmail.com, evellinfisio@hotmail.com, palmiradonda@hotmail.com, arlete.motter@uol.com.br ${ }^{2}$ Autora para correspondência. Complexo Hospital das Clinicas da Universidade Federal do Paraná (Curitiba). Paraná, Brasil. marimar.andreazza@hc.ufpr.br
\end{abstract}

RESUMO | INTRODUçÃO: A posição canguru é uma estratégia do Método Canguru que aproxima as mães de seus filhos, promovendo estabilidade fisiológica. OBJETIVO: Analisar a estabilidade fisiológica e equilíbrio sono-vigília dos RNPTs de uma Unidade de Terapia Intensiva Neonatal (UTIN) em um hospital público universitário, bem como a percepção materna quanto a posição canguru. MÉTODo: Estudo observacional com análise de resultados qualitativos e quantitativos. Desenvolveu-se entre julho a dezembro de 2017 na UTIN. Os dados foram coletados dos prontuários dos RNs. Os parâmetros fisiológicos e estado comportamental de escala sono vigília, antes e após 50 minutos na posição canguru. E as mães responderam à 10 perguntas abertas quanto a seus sentimentos no contato pele a pele. RESULTADOS: Foram incluídos 18 recém-nascidos e 10 mães responderam a entrevista. Os RNs mantiveram a frequência cardíaca $(p=0,28)$ e saturação periférica de oxigênio $(p=0,77)$ nos limites da normalidade, a temperatura corporal apresentou diferença estatisticamente significante $(p=0,01)$ variando entre 36,5 e 36,7 o. Os RNs mudaram o estado comportamental e as mães perceberam estas mudanças durante a realização da posição canguru e se sentiram mais próximas dele por meio do contato pele a pele. CONCLUSÃO: Os recém-nascidos posicionados em canguru no tórax de suas mães mantiveram seus dados fisiológicos estáveis e a temperatura corporal dos mesmos apresentou pequeno acréscimo dentro da normalidade, mostrando ser seguro estar na posição canguru. As mães perceberam que tal ato as aproximou de seus filhos despertando sentimentos positivos de alegria, emoção e amor, indo ao encontro do que é preconizado pelo Método Canguru.

PALAVRAS-CHAVE: Método Canguru. Modalidades de fisioterapia. Recém-nascido. UTI Neonatal.
ABSTRACT | INTRODUCTION: The kangaroo position is a strategy of the Kangaroo Method that brings mothers closer to their children, promoting physiological stability. OBJECTIVE: To analyze the physiological stability and sleep-wake balance of premature newborn from a Neonatal Intensive Care Unit (NICU) in a public university hospital, as well as the maternal perception of the kangaroo position. METHOD: Observational study with analysis of qualitative and quantitative results. It developed between July and December 2017 at the NICU. Data were collected from the newborns' medical records. The physiological parameters and behavioral state of sleep wake scale, before and after 50 minutes in the kangaroo position. And the mothers answered 10 open questions about their feelings about skinto-skin contact. RESULTS: 18 newborns were included and 10 mothers answered the interview. Prelatures maintained their heart rate $(p=$ $0.28)$ and peripheral oxygen saturation $(p=0.77)$ within normal limits, body temperature showed a statistically significant difference $(p=0.01)$ ranging between 36.5 and 36.70. Newborns changed their behavioral state and mothers noticed these changes during the performance of the kangaroo position and felt closer to them through skin-to-skin contact. CONCLUSION: Newborns positioned in a kangaroo on their mothers' thorax kept their physiological data stable and their body temperature showed a small increase within normal limits, showing that it is safe to be in the kangaroo position. The mothers realized that such an act brought them closer to their children, arousing positive feelings of joy, emotion and love, in line with what is recommended by the Kangaroo Method.

KEYWORDS: Kangaroo-Mother Care Method. Physical Therapy Department, Hospital. Newborn. NICU. 


\section{Introdução}

Os avanços tecnológicos na assistência do RN que necessita de cuidados neonatais aumentaram a sobrevida, principalmente de prematuros. Sabe-se que o desenvolvimento adequado desses prematuros é determinado pelo suporte que recebe, seja ele ambiental, familiar e biológico. Toda abordagem do cuidado deve ser continuamente qualificada e revista, pensando no seu adequado desenvolvimento ${ }^{1,2}$.

Com o nascimento prematuro, o desenvolvimento é interrompido e o RN passa a experimentar o ambiente de terapia intensiva que é totalmente diferente do ambiente intrauterino no qual ele se encontrava. Ou seja, se vê exposto a manuseios e procedimentos que podem ser estressantes e dolorosos, além da exposição à luminosidade excessiva e sons altos alterando seu período de sono contínuo, aumentando as experiências não prazerosas nas quais são expostos continuamente ${ }^{3}$.

Nesse contexto, o método canguru, descrito inicialmente em 1978 na Colômbia, surge como uma estratégia alternativa no cuidado de bebês prematuros ou com baixo peso ao nascer. No Brasil, a partir de 2007, esse modelo de cuidado qualificado e humanizado foi integrado à Atenção Humanizada ao Recém-nascido de Baixo Peso e tornou-se parte da política nacional de saúde pública. Faz parte do método o contato pele a pele e propriamente a posição canguru, que consiste em posicionar o recém-nascido na posição vertical junto ao tórax de um dos pais por um tempo máximo, respeitando o familiar e o recém-nascido, de forma segura com orientação de um profissional de saúde capacitado 3 .

Após duas décadas da implantação do Método Canguru no Brasil, há uma vasta evidência científica, nacional e internacional demonstrando as vantagens em sua utilização. Destaca-se a redução do tempo de separação mãe/pai/filho, o que facilita o aleitamento materno, a formação do apego e fortalecimento do vínculo afetivo, além de aumentar a competência e confiança dos pais no cuidado do seu filho, inclusive após a alta hospitalar4.

O RN se beneficia com a posição canguru, na medida em que consegue obter melhor controle térmico, meIhor desenvolvimento neuropsicomotor, redução do estresse e aumento da estimulação sensorial, considerada protetora para um desenvolvimento integral 3 .
Além disso, atua no controle fisiológico, na regulação do estado comportamental, no alívio da dor, o que resulta no aumento da frequência do sono tranquilo dos bebês ${ }^{3 \underline{5}}$ e impacta positivamente na redução da morbimortalidade dos RNs de baixo peso ${ }^{6}$.

Para o RN, além dos benefícios na amamentação, ocorrem também aqueles relacionados à estabilidade fisiológica. O controle térmico está relacionado a idade gestacional e no contato pele a pele com a mãe o RN consegue manter sua temperatura dentro da faixa de normalidade. A frequência cardíaca e a saturação de oxigênio não sofrem efeitos deletérios durante o posicionamento, o sono tranquilo promove melhores taxas de crescimento e mais experiências proprioceptivas positiva ${ }^{\underline{T}}$.

As mães de prematuros experimentam alto índice de estresse e ansiedade no ambiente da UTIN, devido a necessidade de cuidados intensivos e incubadora, atrasando o apego mãe-bebê e a maternagem ${ }^{8}$. Esses eventos podem ser bastante marcantes e até mesmo traumáticos para as mães ${ }^{9}$ causando uma quebra das expectativas idealizadas durante a gestação, acompanhados de sentimentos de angústia e de insegurança ${ }^{10}$.

Diante do exposto, o objetivo do estudo foi analisar a estabilidade fisiológica e equilíbrio sono-vigília dos RNPTs de uma UTIN, bem como a percepção materna quanto a posição canguru.

\section{Método}

Estudo de caráter observacional com análise de resultados quantitativos e qualitativos, realizado na UTIN de um hospital universitário. A coleta de dados foi realizada no período de julho a dezembro de 2017. Este estudo foi conduzido conforme as normas do Comitê de Ética e Pesquisa da instituição, conforme CAAE 71352617.9.0000.0096. Todos os indivíduos que atenderem aos critérios de elegibilidade para inclusão no estudo participaram voluntariamente do mesmo, por meio de permissão de seus pais, os quais assinaram um único termo de consentimento livre e esclarecido. A amostragem foi por conveniência, determinada pelo período de coleta de dados do estudo. Foram incluídos no estudo: Os RNs com até 36 semanas e 6 dias de idade gestacional corrigida que estivessem estáveis hemodinamicamente e 
que a mãe estivesse disponível em realizar a posição canguru por pelo menos 50 minutos. As mães que realizaram posição canguru foram convidadas a responder uma breve entrevista. Foram excluídos os recém-nascidos malformados, sob ventilação mecânica invasiva ou não invasiva e que estivessem em período pós-operatório inferior a sete dias.

Os dados referentes ao nascimento, história materna, processo de tratamento durante internamento e estado de saúde atual foram obtidos dos prontuários.

As observações para registro de parâmetros fisiológicos foram realizadas em dois momentos: antes de serem posicionados, ou seja, ainda na incubadora ou no berço, e após 50 minutos em posição canguru. $O$ tempo foi controlado pelo avaliador por meio de um cronômetro.

Os lactentes, apenas de fralda, foram posicionados em canguru, pelo fisioterapeuta pesquisador, no tórax, também despido, de suas mães, promovendo o contato pele a pele. $O$ dorso do bebê foi coberto com cueiro, mantendo-se assim a posição Canguru por 50 minutos.

Os parâmetros fisiológicos dos neonatos, como a frequência cardíaca e a saturação periférica de oxigênio, foram observados utilizando-se do equipamento disponível na unidade (Monitor multiparamétrico). A temperatura corporal foi aferida com o auxílio de um termômetro axilar e seu estado comportamental de sono e vigília, no que tange às fases do sono e vigília, foi analisado através da escala de avaliação do ciclo de sono e vigília adaptada de Brazelton (1977). Essa escala é constituída por seis classificações intitulados sono profundo, sono leve, sonolência, acordado hipoativo, acordado ativo, choroso, e para cada item, com apenas uma alternativa possível ${ }^{11,12}$. Tais estados foram observados antes e ao final de 50 minutos em posição canguru, e para fins estatísticos foram agrupados dois estados. Foi considerado o estado sono profundo, sono leve e sonolento como dormindo. E acordado, para os estados: alerta calmo, alerta agitado e choro.

A entrevista materna ocorreu após o posicionamento canguru e foi guiada pelo profissional que transcreveu para o questionário as respostas das mães. A partir do referencial teórico de Bardin ${ }^{13}$, foi criada a categoria denominada percepção materna, sendo que a mesma gerou as seguintes subcategorias: sentimentos maternos ao realizar a posição canguru; reconhecimento materno quanto a percepção do estado comportamental no RN. A entrevista teve 10 perguntas fechadas e 5 abertas. Importante ressaltar que nenhuma mãe foi obrigada a responder tal instrumento de pesquisa, a mesma só respondeu por livre e espontânea vontade, e tal questão sempre foi respeitada a todo momento.

Realizou-se a estatística descritiva, as medidas de tendência central e de dispersão estão expressas em médias e desvio padrão para as variáveis contínuas simétricas e em medianas, mínimo e máximo para as assimétricas. Para análise das variáveis contínuas de amostras dependentes foi realizado o Teste T Student para amostras dependentes e teste exato de Fisher para variáveis categóricas. A amostra foi por conveniência delimitada por tempo, foi considerado o nível de significância de $5 \%$, erro do tipo II de 10\% e magnitude do efeito mínimo de $25 \%$, obtendo poder de teste de $90 \%$. A análise qualitativa dos dados baseou-se no referencial teórico de Bardin.

\section{Resultados}

Participaram deste estudo $18 \mathrm{RNs}$, sendo 8 gemelares. Totalizando 14 mães, destas, 4 não quiseram responder à entrevista, concordando somente em realizar a posição canguru, e 10 mães concordaram em responder a entrevista após vivenciarem a posição canguru durante a hospitalização dos seus filhos na UTIN. Os dados maternos estão apresentados na Tabela 1.

Das mães participantes, 7 (70\%) relataram alguma intercorrência durante a gestação. Sendo que as principais intercorrências foram relacionadas à Síndrome de Hellp, infecção urinária, descolamento de placenta, hipertensão, diabetes gestacional, transfusão feto fetal e oligodramnio.

As variáveis fisiológicas coletadas antes do RN ser posicionado em canguru e depois de 50 minutos estão descritas na Tabela 2, não houve diferença na FC e saturação de oxigênio nos dois momentos, entretanto na variável temperatura houve diferença signifcativa $(p=0,01)$, tendo um aumento dentro dos limites da normalidade após o período na posição canguru. 
A mudança de estados comportamentais está representada na Tabela 3. Os RNs que estavam acordados passaram para dormindo decorridos os 50 minutos de posição canguru $(p=0,0034)$.

Quanto à percepção materna, na subcategoria sentimentos maternos ao realizar a posição canguru, nas respostas das mães predominaram os sentimentos positivos, tais como alegria, emoção, amor, realização da maternidade, felicidade, maior proximidade com os filhos, ao mesmo tempo em que também surgiu sentimentos de medo, apreensão e ansiedade. Na subcategoria reconhecimento materno quanto a mudança de estado comportamental do RN, as mães relataram que os RNs passaram de irritado, choroso, agitado, com muitos movimentos desorganizados, para calmo, sono profundo, expressão de tranquilidade e sem movimentos corporais.

Tabela 1. Características maternas

\begin{tabular}{|c|c|c|c|c|}
\hline Variáveis & $\mathrm{n}$ & Percentual & Média & $\pm \mathrm{DP}$ \\
\hline Idade da mãe & 10 & & 31,1 & $\pm 6,64$ \\
\hline \multicolumn{5}{|l|}{ Grau de escolaridade } \\
\hline Ensino Superior Completo & 2 & $20 \%$ & & \\
\hline Ensino Médio Completo & 8 & $80 \%$ & & \\
\hline \multicolumn{5}{|l|}{ Estado civil } \\
\hline Casada & 9 & $90 \%$ & & \\
\hline Solteira & 1 & $10 \%$ & & \\
\hline \multicolumn{5}{|l|}{ Número de gestações } \\
\hline $1^{\mathrm{a}}$ gestação & 4 & $40 \%$ & & \\
\hline $2^{a}$ gestação & 2 & $20 \%$ & & \\
\hline $3^{\mathrm{a}}$ gestação & 3 & $30 \%$ & & \\
\hline $4^{a}$ gestação & 1 & $10 \%$ & & \\
\hline Realizou pré-natal & 10 & $100 \%$ & & \\
\hline Número de consultas & & & 7 & $\pm 2,7$ \\
\hline Intercorrência durante gestação* & 7 & $70 \%$ & & \\
\hline
\end{tabular}

Notas:DP: Desvio padrão

*Intercorrências durante a gestação:Síndrome de Hellp, infecção urinária, descolamento de placenta, hipertensão, diabetes gestacional, transfusão feto fetal e oligodramnio.

Tabela 2. Variáveis do recém-nascido antes e depois de 50 minutos na posição canguru

\begin{tabular}{lrrr}
\hline & \multicolumn{1}{c}{ Antes } & \multicolumn{1}{c}{ Depois } & $\mathrm{p}$ \\
\hline Frequência Cardíaca & $143,4( \pm 11,76)$ & $146,3( \pm 12,2)$ & 0,28 \\
Saturação Oxigênio & $95,2( \pm 3,11)$ & $95,4( \pm 2,81)$ & 0,77 \\
Temperatura axilar & $36,59( \pm 0,24)$ & $36,76( \pm 0,27)$ & 0,01 \\
\hline
\end{tabular}

Teste estatístico: Teste T de Student

Tabela 3. Estados Comportamentais dos recém-nascidos

\begin{tabular}{c|c|c|c}
\hline Momento & Acordado & Dormindo & $\mathrm{p}$ \\
\hline Antes & $13(72 \%)$ & $5(28 \%)$ & 0,0034 \\
Depois & $4(22 \%)$ & $14(78 \%)$ & \\
\hline
\end{tabular}

Nota: Teste estatístico: Teste de Exato de Fisher 


\section{Discussão}

Os dados fisiológicos dos RNs se mantiveram estáveis durante a posição canguru, e suas mães sentiram emoção e alegria por estarem mais próximas a eles. Estas mães são adultas jovens, o que, segundo a literatura, é um fator protetor contra a prematuridade. Em estudo realizado para analisar a influência da idade materna com os desfechos perinatais em gestações de alto risco, verificou-se que mães muito jovens possuem chances maiores de terem filhos com BPN, além de baixo escore de Apgar e óbito neonatal. Assim como, gestantes com idade acima de 36 anos, tem maiores chances de terem filhos prematuro ${ }^{14}$.

Quanto à escolaridade, a maioria das mães participantes do estudo tinham ensino médio completo, o que é positivo, visto que a baixa escolaridade é condição que pode predispor a situações potencialmente de risco para a mãe e o recém-nascido, além de impedir o acesso a informações e orientações, restringir a capacidade de cuidado e assistência, podendo levar inclusive ao início tardio ou ausência ao pré-natal ${ }^{15}$.

Chamou atenção a quantidade de mães que relataram alguma intercorrência durante a gestação (70\%). Estes achados são consonantes com os demonstrados em pesquisas anteriores, cuja finalidade foi caracterizar o perfil sociodemográfico e fatores de risco materno de RNPTs hospitalizados em UTIN 16 .

O contato pele a pele se mostra eficaz e seguro como método analgésico para a dor do recém-nascido ${ }^{17}$. Por outro lado, o controle da dor em neonatos prematuros contribui para a estabilidade clínica e diminui complicações. Entre as medidas não farmacológicas propostas estão o posicionamento adequado, contenção, uso de substâncias adocicadas, sucção, posição canguru, controle sonoro, térmico e de luminosidade do ambiente ${ }^{18}$.

Os recém-nascidos observados neste estudo mantiveram a frequência cardíaca e saturação dentro dos limites da normalidade, mostrando que houve estabilidade entre o antes e depois do posicionamento. Houve uma diferença significativa na temperatura corporal, ( $p, 01)$ corroborando com a proposta do método que é de estabilizar a temperatura do recém-nascido prematuro por meio do contato pele a pele 3 .
Um estudo iraniano comparou um grupo experimental e um grupo controle. Para ambos os grupos, os parâmetros fisiológicos do bebê foram medidos três vezes em cada prática da posição canguru (antes, 15 minutos após o início da posição e após a posição canguru). Após a posição canguru, o grupo experimental apresentou diferença significativa nos parâmetros fisiológicos estudados (frequência cardíaca, saturação periférica de oxigênio e temperatura corporal $)^{19}$.

No estudo de Tenório et al., (2010) as variáveis frequência cardíaca, saturação periférica de oxigênio e temperatura corporal, peso corporal e pressão arterial média foram avaliadas antes e 30 minutos após a posição canguru. Previamente a intervenção, todos os bebês foram avaliados em decúbito dorsal, utilizando somente fraldas, na incubadora ou berço aquecido. Foram observadas melhoras significativas na frequência cardíaca, saturação periférica de oxigênio, temperatura corporal e pressão arterial média, enquanto que as variáveis frequência cardíaca e peso corporal não apresentaram diferenças significativas ${ }^{20}$.

Neste estudo foi observado pela escala de Brazelton e também pela percepção materna que os RNs estavam acordados se movimentando espontaneamente e alguns chorosos enquanto estavam no berço e ao final de 50 minutos em posição canguru mudaram de estado comportamental para dormindo, calmos e com pouca movimentação ativa .

Sabe-se que o ambiente das UTINs oferece aos RNs muitos estímulos deletérios, como ruídos, iluminação intensa, muitas manipulações, consequentemente, alterando os ciclos de sono-vigília tão importantes ao desenvolvimento neurológico adequado ${ }^{21}$. Proporcionar ao RN momentos onde ele possa entrar em sono profundo, protegido dos fatores ambientais e no aconchego de seus pais é um dos objetivos preconizado pelo método canguru ${ }^{3}$.

Os ciclos de sono no feto variam de 40 a 60 minutos e RNs a termo dormem $70 \%$ do dia apontando para uma forte relação entre sono e crescimento, incluído cerebral22. É um desafio para as equipes de assistência saber diferenciar os estados de sono vigília oferecer ao RN estratégias que proporcionem conforto e sono de qualidade, pois a privação de sono tem um impacto negativo para o desenvolvimento neuronal, alterações da respiração e pode contribuir para aumento das morbidades 23 . 
As mães também se beneficiam do contato pele a pele com seus RNs, tendo impacto positivo sobre a dor materna após o parto, e diminuição do estresse, avaliado pelo cortisol salivar ${ }^{24}$. Além disso, a presença paterna tem representado um suporte físico e emocional para a mãe, proporcionando conforto, segurança e felicidade, com repercussão positiva para a amamentação. O cuidado do pai, com a mãe e com o filho prematuro gera satisfação para a mãe, devendo esta prática ser valorizada pelas unidades de atendimento aos RNs hospitalizados 25,26 .

As mães participantes deste estudo, mostraram-se felizes e animadas com a possibilidade de realizar a posição canguru com seus bebês e entre os principais benefícios citados por elas estão: saúde dos RNs, aumento do vínculo, ganho de peso e desenvolvimento dos seus filhos prematuros. Esses resultados corroboram com o estudo de Castanhade et al (2020) no qual as mães relataram benefícios imunológicos, ganho de peso, controle da temperatura e afetividade ${ }^{27}$.

A literatura tem demonstrado que as mães adquirem segurança e autonomia para cuidar do bebê, na medida em que dispõem de um pronto acesso a seus filhos e são orientadas e incentivadas a realizar o contato pele a pele, evoluindo a partir do toque até a posição canguru, bem como, os cuidados progressivos e de forma segura, desde a troca de fralda, realização do banho, e administração da alimentação, seja por sonda, pelo copo ou amamentação. Assim, o Método Canguru promove a participação dos pais e fortalece a relação de vínculo afetivo entre mãe/filho ${ }^{28}$. 0 número reduzido de mães entrevistadas pode representar uma limitação deste estudo, assim, sugere-se pesquisas futuras para atenuar este aspecto.

\section{Conclusão}

Refletir sobre posicionar os recém-nascidos com segurança, sabendo que seus dados vitais continuarão estáveis na posição canguru, valida o método na unidade estudada, trazendo para a equipe conforto e confiança para servirem de facilitadores na relação entre as mães e seus filhos. Apesar do número reduzido de participantes, foi possível observar que os recém-nascidos mantiveram a frequência cardíaca e saturação de oxigênio sem alterações entre o antes e o depois do posicionamento, e a temperatura teve crescimento com diferença estatisticamente significativa. As mães expressaram verbalmente a sensação positiva que tiveram após permanecer com seus bebês na posição canguru, e perceberam que seus filhos ficaram mais tranquilos e dormiram.

\section{Contribuições das autoras}

Nisi KSA participou da concepção, delineamento, coleta de dados e revisão do manuscrito. Andreazza MG participou da concepção, delineamento, busca e análise estatística dos dados da pesquisa, interpretação dos resultados e redação do artigo científico. Gomes EO participou da coleta e análise estatística dos dados da pesquisa, interpretação dos resultados e redação do manuscrito. Soares PD participou da coleta de dados, interpretação dos resultados e redação do manuscrito. Motter AA participou da concepção, delineamento, coleta de dados, interpretação dos resultados, redação do manuscrito e orientação da pesquisa.

\section{Conflitos de interesses}

Nenhum conflito financeiro, legal ou político envolvendo terceiros (governo, empresas e fundações privadas, etc.) foi declarado para nenhum aspecto do trabalho submetido (incluindo, mas não se limitando a subvenções e financiamentos, participação em conselho consultivo, desenho de estudo, preparação de manuscrito, análise estatística, etc.).

\section{Referências}

1. Otoni ACS, Grave MTQ. Avaliação dos sinais neurocomportamentais de bebês pré-termo internados em Unidade de Terapia Intensiva Neonatal. Rev Ter Ocup Univ São Paulo. 2014;25(2):151-8. doi: 10.11606/issn.2238-6149. v25i2p151-8

2. Lamy ZC, Gomes MASM, Gianini NOM, Hennig MAS. Atenção humanizada ao recém-nascido de baixo peso - Método Canguru: a proposta brasileira. Cien Saude Colet. 2005;10(3):659-668. doi: 10.1590/S1413-81232005000300022

3. Ministério da Saúde. Atenção Humanizada ao Recém - Nascido: Método Canguru, Manual Técnico [Internet]. 2017. Disponível em: http://bvsms.saude.gov.br/bvs/publicacoes/atencao_humanizada_ recem_nascido_canguru.pdf 
4. Zirpoli DB, Mendes RB, Reis TS, Barreiro MSC, Menezes AF. Benefícios do Método Canguru: Uma Revisão Integrativa. J Res Fundam Care. 2019;11(2):547-54. doi: 10.9789/2175-5361.2019. v11i2.547-554

5. Lotto CR, Linhares MBM. Contato "pele a pele" na prevenção de dor em bebês prematuros: revisão sistemática da literatura. Trends Psychol. 2018;26(4):1699-713. doi: 10.9788/tp2018.4-01pt

6. Conde-Agudelo A, Díaz-Rossello JL. Kangaroo mother care to reduce morbidity and mortality in low birthweight infants. Cochrane Database of Systematic Reviews. 2016;2016(8)11-21. doi: 10.1002/14651858.CD002771.pub4

7. Kirsten GF, Bergman NJ, Hann FM. Kangaroo mother care in the nursery. Pediatr Clin North Am. 2001;48(2):443-52. doi: 10.1016/ s0031-3955(08)70036-1

8. Badiee Z, Faramarzi S, MiriZadeh T. The effect of kangaroo mother care on mental health of mothers with low birth weight infants. Adv Biomed Res. 2014;3:214. doi: 10.4103/2277. 9175.143262

9. Heck GMM, Lucca HC, Costa R, Junges CF, Santos SV, Borck M. Compreensão do sentimento materno na vivência no método canguru. Rev Enferm UFSM. Universidad Federal de Santa Maria; 2016;6(1):71-83. doi: 10.5902/2179769218083

10. Baseggio DB, Dias MPS, Brusque SR, Donelli TMS, Mendes P. Vivências de mães e bebês prematuros durante a internação neonatal. Temas Psicol. 2017;25(1):153-67. doi: $\underline{10.9788 /}$ TP2017.1-10

11. Barbosa M, Moreira J, Tronick E, Beeghly M, Fuertes M. Neonatal Behavioral Assessment Scale (NBAS): Confirmatory factor analysis of the six behavioral clusters. Early Hum Dev. 2018;124:1-6. doi: 10.1016/j.earlhumdev.2018.07.007

12. Brazelton TB, Nugent JK. Neonatal Behavioral Assessment Scale. Clinics in Developmental Medicine: Mac Keith Press;1973.

13. Bardin L. Análise de Conteúdo (1 a ed). São Paulo: Edições 70; 2016. 279 p.

14. Almeida LIV, Ramos SB, Figueiredo GLA. Apoio e rede social no contexto urbano: percepções de mães de crianças prematuras. Aletheia. 2019;52(1):22-36.

15. Ramos HÂC, Cuman RKN. Fatores de risco para prematuridade: pesquisa documental. Esc Anna Nery. 2009;13(2):297-304. doi: 10.1590/\$1414-81452009000200009

16. Carvalho SS, Oliveira BR. Perfil epidemiológico de puérperas e recém-nascidos atendidos em uma unidade de referência. Rev Saúde Col UEFS. 2019;9(0):159. doi: 10.13102/rscdauefs.v9.4493
17. Maciel HIA, Costa MF, Costa ACL, Marcatto JO, Manzo BF, Bueno M. Pharmacological and nonpharmacological measures of pain management and treatment among neonates. Rev Bras Ter Intensiva. 2019;31(1):21-6. doi: 10.5935/0103-507x.20190007

18. Andreazza MG, Motter AA, Cat ML, Silva RPGVC. Percepção da dor em neonatos pela equipe de enfermagem de unidade de terapia intensiva neonatal. Rev Bras Pesq Saúde. 2017;19(4):1339. doi: 10.21722/rbps.v19i4.19813

19. Parsa P, Karimi S, Basiri B, Roshanaei G. The effect of kangaroo mother care on mental health of mothers with premature infants. Pan Afr Med J. 2018;30(89):1-8. doi: 10.11604/ pamj.2018.30.89.14428

20. Tenório EAM, Mota GC, Gutierres SB, Ferreira ERS, Medeiros AA, Tavares CAE et al. Avaliação dos parâmetros fisiológicos em recémnascidos pré-termos de baixo peso antes e após a aplicação do método mãe-canguru TT. Fisioter Bras. 2010;11(1):44-8.

21. Gomes ELFD, Santos CM, Santos ACS, Silva AG, França MAM, Romanini DS et al. Respostas autonômicas de recém-nascidos prematuros ao posicionamento do corpo e ruídos ambientais na unidade de terapia intensiva neonatal. Rev Bras Ter Intensiva. 2019;31(3):296-302. doi: 10.5935/0103-507x.20190054

22. Montemitro E, Franco P, Scaillet S, Kato I, Groswasser J, Villa MP et al. Maturation of spontaneous arousals in healthy infants. Sleep. 2008;31(1):47-54. doi: 10.1093/sleep/31.1.47

23. Bonan KCSC, Pimentel Filho JC, Tristão RM, Jesus JAL, Campos Junior D. Sleep deprivation, pain and prematurity: a review study. Arq Neuro-Psiquiatr. 2015;73(2):147-54. doi: 10.1590/0004$\underline{282 \times 20140214}$

24. Kollmann M, Aldrian L, Scheuchenegger A, Mautner E, Herzog SA, Urlesberger B et al. Early skin-to-skin contact after cesarean section: A randomized clinical pilot study. PLoS One. 2017;12(2): e0168783. doi: 10.1371/journal.pone.0168783

25. Santana JO, Borges KI, Souza DA, Pinto KRTF, Rossetto EG, Zani AV. O Cuidado Paterno Ao Filho Prematuro Hospitalizado: Representações Maternas. Rev Baiana Enferm. 2018;31(4):1-8. doi: 10.18471/rbe.v31i4.22310

26. Hagen IH, Iversen VC, Svindseth MF. Differences and similarities between mothers and fathers of premature children: A qualitative study of parents' coping experiences in a neonatal intensive care unit. BMC Pediatrics. 2016;16:92. doi: $10.1186 /$ s12887-016-0631-9

27. Cantanhede ES, Amorim FCM, Oliveira ADS, Almeida CAPL, Santos SM. Experiências das mães no cuidado ao recémnascido prematuro no método canguru. Cogitare Enferm. 2020; 25:e67416. doi: $10.5380 / c e . v 25 i 0.67416$

28. Silva JMQ, Paiva MS, Almeida MS. Significado para as mães sobre a vivência no Método Canguru [dissertação]. Salvador: Universidade Federal da Bahia - Escola de Enfermagem; 2014. 